\title{
1 A plant pathogen utilizes effector proteins for microbiome manipulation
}

2

3 Nick C. Snelders ${ }^{1}$, Hanna Rovenich ${ }^{1, \#}$, Gabriella C. Petti ${ }^{1,2, \#}$, Mercedes Rocafort ${ }^{1, \neq}$, Julia A. Vorholt ${ }^{2}$,

4 Jeroen R. Mesters ${ }^{3}$, Michael F. Seidl ${ }^{1,4}$, Reindert Nijland ${ }^{1, \S}$, Bart P.H.J. Thomma ${ }^{1,5, *}$

5

$6{ }^{1}$ Laboratory of Phytopathology, Wageningen University \& Research, Wageningen, The Netherlands

$7 \quad{ }^{2}$ Institute of Microbiology, ETH Zürich, Zürich, Switzerland

$8{ }^{3}$ Institute of Biochemistry, University of Lübeck, Center for Structural and Cell Biology in Medicine,

9 Lübeck, Germany

$10{ }^{4}$ Theoretical Biology \& Bioinformatics Group, Department of Biology, Utrecht University, Utrecht, The

11 Netherlands

$12{ }^{5}$ Cluster of Excellence on Plant Sciences (CEPLAS), University of Cologne, Botanical Institute, Cologne,

13 Germany

15 "These authors contributed equally

$16{ }^{\ddagger}$ Current address: School of Agriculture and Environment, Massey University, Palmerston North, New

17 Zealand

$18{ }^{\S}$ Current address: Marine Animal Ecology Group, Wageningen University \& Research, Wageningen, 19 The Netherlands

*Corresponding author. Email: bart.thomma@wur.nl 


\section{Abstract}

During colonization of their hosts, pathogens secrete effector proteins to promote disease development through various mechanisms. Increasing evidence shows that the host microbiome plays a crucial role in health, and that hosts actively shape their microbiomes to suppress disease. We hypothesized that pathogens evolved to manipulate host microbiomes to their advantage in turn. Here, we show that the fungal plant pathogen Verticillium dahliae utilizes effector proteins for niche colonization through selective manipulation of host microbiomes by suppressing microbes with antagonistic activities. Moreover, we show that effector proteins are similarly exploited for microbiome manipulation in the soil environment, where the fungus resides in absence of a host. In conclusion, we demonstrate that pathogens utilize effector proteins to modulate microbiome compositions and propose that their effector catalogs represent an untapped resource for novel antibiotics.

\section{Introduction}

To establish disease, pathogenic microbes secrete a wide diversity of effector proteins that facilitate host colonization through a multitude of mechanisms ${ }^{1}$. Typically, pathogen effectors are defined as small cysteine-rich proteins that are secreted upon colonization to manipulate host physiology or to deregulate host immune responses ${ }^{2}$. Consequently, effector proteins are predominantly studied in binary host-microbe interactions, while largely ignoring the biotic context in which these interactions take place. Higher organisms, including plants, associate with a plethora of microbes that collectively form their microbiome, which represents a key determinant for their health ${ }^{3-7}$. The most extensive microbial colonization of plants occurs at roots, where plants define rhizosphere microbiome compositions through secretion of exudates $s^{8,9}$ and specifically attract beneficial microbes to suppress pathogen invasion ${ }^{10-12}$. Thus, we hypothesized that plant pathogens evolved mechanisms to counteract this recruitment and modulate host microbiomes for successful infection, possibly through effector proteins ${ }^{1,13}$. 
Verticillium dahliae is a soil-borne fungus that causes vascular wilt disease on hundreds of plant species, including numerous $\operatorname{crops}^{14,15} . V$. dahliae survives in the soil through persistent resting structures called microsclerotia that germinate in response to nutrient-rich exudates released by nearby plant roots ${ }^{16}$. Subsequently, emerging hyphae grow through the soil and rhizosphere towards the roots where the fungus penetrates its hosts. Following root penetration, $V$. dahliae invades the xylem where it produces conidiospores that are spread throughout the vasculature by the sap stream. This systemic colonization causes chlorosis and necrosis of plant tissues, which is followed by plant senescence. $V$. dahliae then enters a saprophytic phase, emerges from the vasculature and colonizes the dead plant material where it produces new microsclerotia that are eventually released into the soil upon tissue decomposition.

Using comparative population genomics, we previously identified the $V$. dahliae-secreted small cysteine-rich effector protein Ave1 that is recognized as an avirulence determinant by tomato plants that carry the corresponding Ve1 immune receptor ${ }^{17}$. However, on host plants lacking Ve1, VdAve1 acts as a virulence effector that promotes fungal colonization and disease development ${ }^{17}$. Interestingly, VdAve1 is homologous to plant natriuretic peptides (PNPs) that have been identified in numerous plant species, suggesting that VdAve1 was acquired from plants through horizontal gene transfer ${ }^{17}$. Whereas several of the plant PNPS were shown to act in plant homeostasis and (a)biotic stress responses $^{18,19}$, the mode of action of $\mathrm{VdAve} 1$ to contribute to fungal virulence has remained unknown.

Intriguingly, unlike most pathogen effector genes characterized to date, VdAve1 is not only highly expressed during host colonization ${ }^{17,20}$, but also during growth in vitro and under conditions mimicking soil colonization, suggesting a ubiquitous role throughout the fungal life cycle including life stages outside the host, and thus a role that does not primarily involve targeting host plant physiology (Extended data Fig. 1). Our attempts to purify VdAve1 upon heterologous expression in Escherichia coli, to facilitate functional characterization, repeatedly failed due to the formation of 
inclusion bodies (Extended data Fig. 2a). The inability to obtain soluble protein using heterologous microbial expression systems can be attributed to a multitude of reasons, but is a well-known phenomenon when expressing antimicrobial proteins ${ }^{21}$. Consequently, based on the ubiquitous expression of $V d A v e 1$ by $V$. dahliae, and our inability to purify soluble $V d A v e 1$ following expression in E. coli, we hypothesized that $\mathrm{VdAve} 1$ may possess antimicrobial activity.

To obtain functional VdAve1, inclusion bodies were isolated from E. coli cells and denatured using guanidine hydrochloride. Next, VdAve1 was refolded by stepwise dialysis and functionality was confirmed through testing recognition by its immune receptor Ve1 (Extended data Fig. 2b). To assess the potential antimicrobial activity of VdAve1, we developed an in vitro system in which we incubated a panel of plant-associated bacteria in tomato xylem fluid, to mimic a natural environment in which VdAve1 is secreted, namely tomato xylem vessels, and monitored their growth in presence and absence of the protein. Interestingly, VdAve1 selectively inhibited the growth of plant-associated bacteria (Fig. 1a). Whereas growth of all Gram positive bacteria tested, namely Arthrobacter sp., Bacillus subtilis, Staphylococcus xylosus and Streptomyces sp., was strongly inhibited, Gram negative bacteria displayed differential sensitivity to the protein. Intriguingly, this differential sensitivity is not immediately explained by phylogenetic relationships of the tested isolates as even within bacterial orders/families differences are observed. For instance, whereas growth of the burkholderiales species Acidovorax is inhibited by VdAve1, growth of a Ralstonia isolate, which belongs the same order, is not. Similarly, treatment of two closely related rhizobiales, Rhizobium sp. and Agrobacterium tumefaciens, revealed differential sensitivity as VdAve1 affected growth of Rhizobium sp., but not of $A$. tumefaciens. Finally, growth of Pseudomonas corrugata and Serratia sp. was only slightly altered and unaffected, respectively, while growth of both Sphingobacterium sp. and Sphingomonas mali was affected upon exposure to VdAve1. Interestingly, growth of the endophytic fungus Fusarium oxysporum and the fungal mycoparasite Trichoderma viride was not inhibited by VdAve1, suggesting that VdAve1 exerts antibacterial, but not antifungal, activity (Extended data Fig. 
bioRxiv preprint doi: https://doi.org/10.1101/2020.01.30.926725; this version posted January 31,2020 . The copyright holder for this preprint (which was not certified by peer review) is the author/funder, who has granted bioRxiv a license to display the preprint in perpetuity. It is made available under aCC-BY 4.0 International license.

$973 a)$. These initial observations with divergent, randomly chosen, plant-associated bacteria prompted

98 us to further characterize the antimicrobial activity of VdAve1.

a
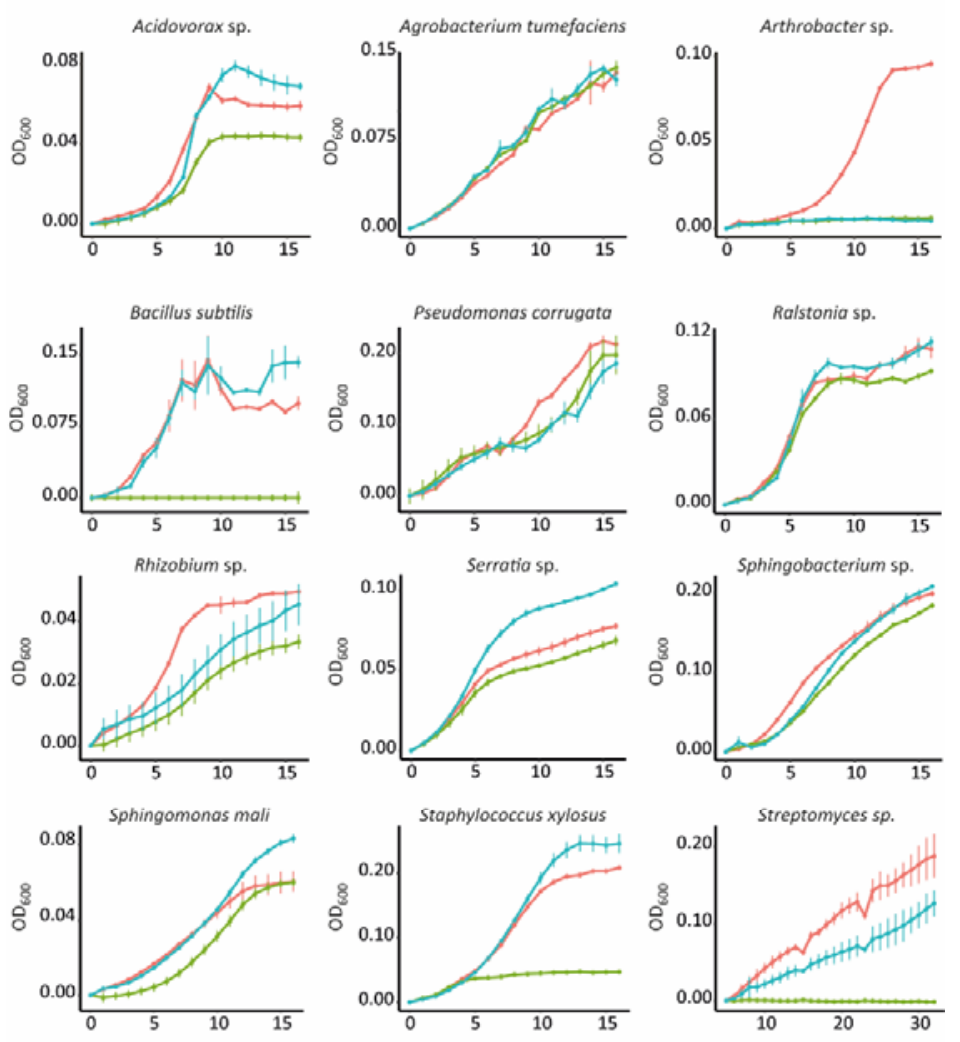

b

$$
+ \text { MQ }+8 \mu \text { MVdAve1 }+8 \mu M \text { VnAve1 }
$$

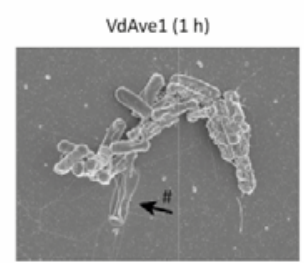

VdAve1 $(4 \mathrm{~h})$

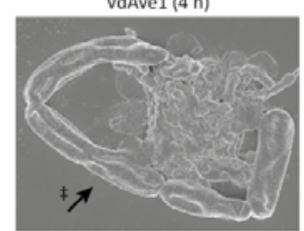

VdAve1 (2 h)

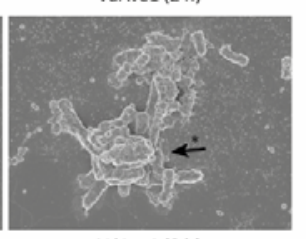

VdAve1 $(8 \mathrm{~h})$

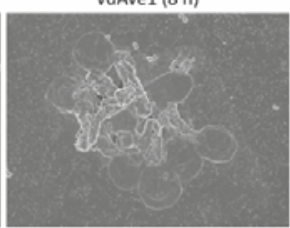

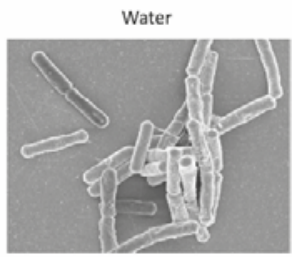

VnAve1 (8 h)

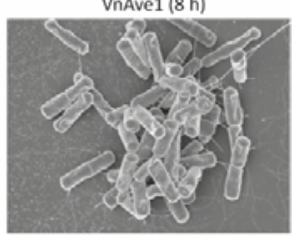

$2 \mu \mathrm{m}$

Fig. 1: Bactericidal activity of Verticillium dahliae effector VdAve1. a, VdAve1 selectively inhibits in vitro growth of plant-associated bacterial isolates in tomato xylem fluid. The close homolog VnAve1 from $V$. nubilum only inhibits a subset of the bacteria affected by VdAve1 and is generally less effective. b, Scanning electron microscopy of $B$. subtilis upon 1, 2, 4 and 8 hours of incubation in tomato xylem fluid showing blebbing $\left({ }^{*}\right)$, swelling (‡) and lysis (\#) with $6.5 \mu \mathrm{M}$ VdAve1 (0.8 x MIC), but not with water or VnAve1. 
As a first step in the further characterization of the antimicrobial activity of VdAve1, we aimed to determine whether the effector protein is bacteriostatic or bactericidal by making use of electron microscopy to visualize the effect of protein treatment on bacteria. As a target species the Gram positive $B$. subtilis was chosen, considering its high sensitivity to VdAve1 treatment. By testing a concentration series of the VdAve1 effector protein, the minimum inhibitory concentration (MIC) was determined at $8 \mu \mathrm{M}$ (Extended data Fig. 3b). However, electron microscopy analysis revealed that sub-MIC concentrations of VdAve1 already induced blebbing and swelling of bacterial cells, followed by lysis and collapse, corresponding with bactericidal activity (Fig. 1b).

To investigate whether the antimicrobial activity that is displayed by VdAve1 is more widely conserved among its homologs, we tested the only homolog that occurs in one of the sister species of the Verticillium genus, namely VnAve1, from the non-pathogenic species $V$. nubilum that displays 90\% amino acid identity (Extended data Fig. 3c). Interestingly, also this homolog displays antimicrobial activity, albeit that it only inhibits a subset of the bacteria affected by VdAve1, and does not cause $B$. subtilis lysis (Fig. 1). Thus, the 13 amino acid polymorphisms between the two Ave1 homologs are responsible for differences in the activity spectrum. To investigate whether the antimicrobial activity also occurs among plant homologs, or is confined to microbial homologs and involves neofunctionalization after horizontal transfer, the more distant homolog AtPNP-A from Arabidopsis thaliana was tested as well. Intriguingly, AtPNP-A completely arrests B. subtilis growth (Extended data Fig. 3c,d). Collectively, these findings demonstrate that various Ave1 homologs possess antimicrobial activity, yet with divergent activity spectra, and suggest that the antimicrobial activity of VdAve1 did not result from neofunctionalization following horizontal gene transfer.

Based on the strong but selective bactericidal activity of VdAve1 in vitro, we hypothesized that $V$. dahliae exploits its effector protein to affect host microbiome compositions through the suppression of other microbes. Therefore, to determine the biological relevance of the observed bactericidal activity, we performed bacterial community analysis based on 165 ribosomal DNA profiling of tomato 
and cotton root microbiomes following infection with wild-type $V$. dahliae or a VdAve1 deletion mutant. Importantly, root microbiome compositions were determined during early $V$. dahliae infection stages, namely at ten days post inoculation when the fungus has just entered xylem vessels and initiated systemic spreading, to minimize indirect shifts in microbial compositions that result from severe disease symptomatology, rather than from direct shifts due to the presence of the effector protein. We did not observe major shifts in overall composition of bacterial phyla (Extended data Fig. 4a) or total microbial diversity ( $\alpha$-diversity) (Extended data Fig. 4b) upon $V$. dahliae colonization of tomato and cotton. However, principal coordinate analysis based on Bray-Curtis dissimilarities ( $\beta$-diversity) revealed a clear separation of root microbiomes (Fig. 2a) (PERMANOVA, $p<0.01$ for both tomato and cotton). Importantly, the extent of $V$. dahliae colonization does not seem to determine the separation, as clustering of $V$. dahliae genotypes occurs in cotton although VdAve1 deletion hardly affects fungal virulence on this host plant (Fig. 2a). Thus, as anticipated based on the potent, yet selective, antimicrobial activity, VdAve1 secretion by $V$. dahliae sophistically alters root microbiome compositions. Arguably, based on the sophisticated effects, a full and detailed characterization of microbiome composition changes requires large sample sizes and abundant numbers of repeats. However, strikingly, despite the relatively small sample size of our 165 rDNA profiling, pairwise bacterial order comparisons upon colonization by wild-type $V$. dahliae and the VdAve1 deletion mutant revealed differential abundances of Sphingomonadales, Bdellovibrionales and Ktedonobacterales for tomato. (Fig. 2b) (Extended data Table 1). The finding that Sphingomonadales are repressed in the presence of $\mathrm{VdAve} 1$ suggests that this taxon is the most sensitive to VdAve1 activity. A similar comparison for cotton did not immediately reveal any differentially abundant orders, but agglomeration of amplicon sequence variants (ASVs) based on phylogenetic relatedness (patristic distance<0.1) revealed eight differentially abundant taxa, including a taxon of the Sphingomonadaceae family (Fig. 2b) (Extended data Table 2). Interestingly, although this taxon only represents a small proportion of all Sphingomonadaceae in the cotton root microbiomes, it is exclusively and consistently found in the microbiomes of roots infected by the 
a
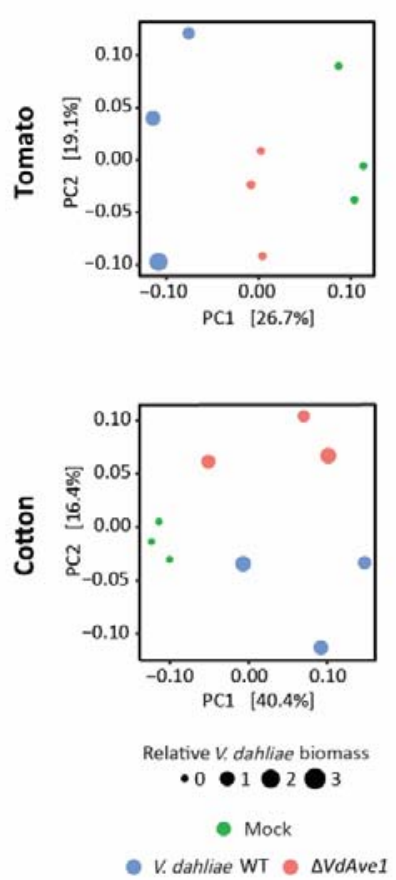

b
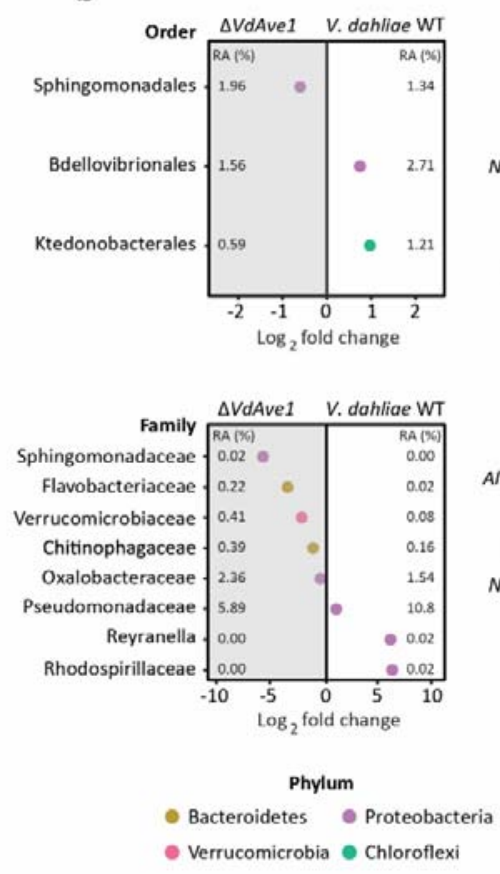

C
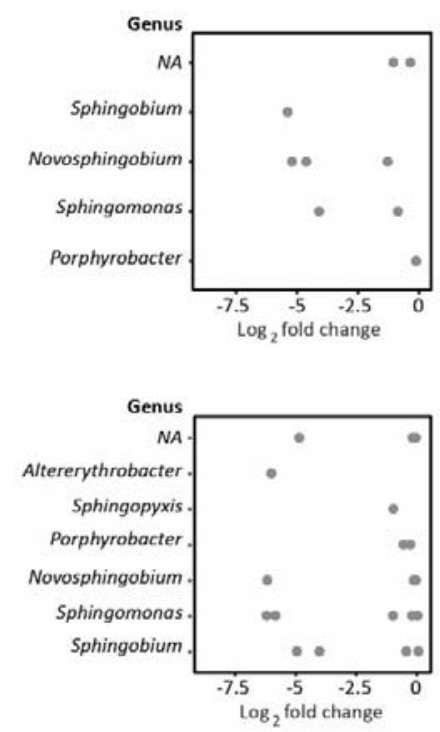

162

163

Fig. 2: Verticillium dahliae VdAve1 impacts root microbiomes. a, Principal coordinate analysis based on Bray-Curtis dissimilarities reveals separation of root microbiome compositions ten days after inoculation with wild-type $V$. dahliae and a VdAve1 deletion mutant (PERMANOVA, $p<0.01$ ). b, Differential abundance analysis of bacterial orders (tomato) and upon agglomeration of amplicon sequence variants (patristic distance<0.1) (cotton) through pairwise comparison between root microbiomes colonized by wild-type $V$. dahliae and a VdAve1 deletion mutant (Wald test, $\mathrm{p}<0.01$ ). The average relative abundance (RA) of the differentially abundant taxa is indicated as a percentage of the total bacterial community in the corresponding root microbiome. c, Sphingomonads (Sphingomonas, Novosphingobium, Sphingopyxis, and Sphingobium) are repressed by VdAve1. Dots represent single amplicon sequence variants with increased abundance (average of 3 samples) in 
root microbiomes upon colonization by the VdAve1 deletion mutant when compared with wild-type V. dahliae.

Given the fact that secretion of VdAve1 by $V$. dahliae during colonization of both tomato and cotton leads to a reduction of Sphingomonadales in the corresponding root microbiomes, we anticipated a broad efficacy of VdAve1 on bacteria within this order. Therefore, to identify Sphingomonadales genera that are most sensitive to VdAve1, we identified ASVs with increased average relative abundance in the microbiomes with the VdAve1 deletion mutant when compared with wild-type $V$. dahliae, revealing Sphingomonas, Novosphingobium, Sphingopyxis and Sphingobium that are commonly referred to as Sphingomonads (Fig. $2 \mathrm{c})^{22,23}$. To confirm that the reduced Sphingomonad abundance during $V$. dahliae colonization is a direct consequence of VdAve1 activity, we tested the sensitivity of a panel of plant-associated Sphingomonads to VdAve1 in vitro ${ }^{24,25}$. In accordance with the previously observed effect on S. mali (Fig. 1a), treatment with VdAve1 was found to also inhibit growth of Sphingobium, Novosphingobium, Sphingopyxis and two other Sphingomonas species (Fig. 3a), indicating a broad sensitivity among the Sphingomonads. Given the selective efficacy of VdAve1 and the strong effect on Sphingomonads in the tomato and cotton microbiomes, we hypothesized that these bacteria may act as antagonists and negatively affect $V$. dahliae growth in the absence of VdAve1. Indeed, co-cultivation of $V$. dahliae with Novosphingobium sp. A and S. macrogoltabida resulted in reduced fungal biomass for the VdAve1 deletion mutant, when compared with the $V$. dahliae wild-type that secretes VdAve1 under these conditions, revealing that Sphingomonads comprise antagonists of $V$. dahliae, and explaining the importance of their inhibition by VdAve1 (Fig. 3b). Accordingly, and in line with previously described observations of plant protective activities of Sphingomonad strains ${ }^{24}$, pre-treatment of surface-sterilized tomato seeds with S. macrogoltabida negatively affected Verticillium wilt disease development as confirmed through biomass quantification of wild-type $V$. dahliae in the presence and the absence of the bacterium (Fig. 3c,d) (Extended data Fig. 5). Importantly, quantification of S. macrogoltabida in the presence of wild-type V. dahliae and the VdAve1 deletion mutant using 165 rDNA profiling and real-time PCR revealed that VdAve1 secretion significantly impacts $S$. macrogoltabida proliferation to counter its protective effect 
201 (Fig. 3e-g). Notably, this observation is not an indirect effect of differential host colonization by wild-

202 type $V$. dahliae and the VdAve1 deletion mutant, as selection of tomato plants with equal levels of $V$.

203 dahliae biomass (Fig. 3d, data points highlighted in red), reveals similarly impaired S. macrogoltabida

proliferation in the presence of $\mathrm{VdAve1}$ (Fig. $3 \mathrm{~g}$ ). Thus, these data underpin the hypothesis that $V$. during host colonization.

a
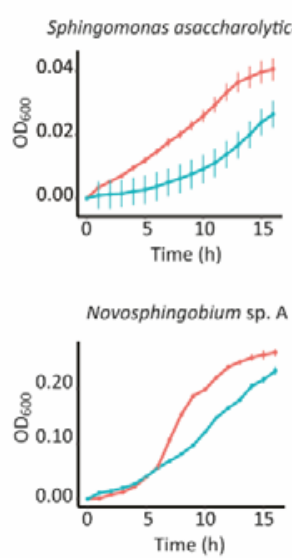

C

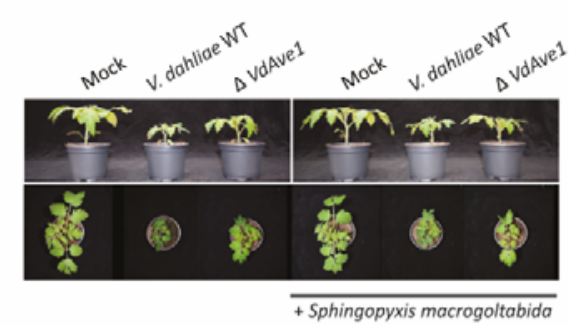

d
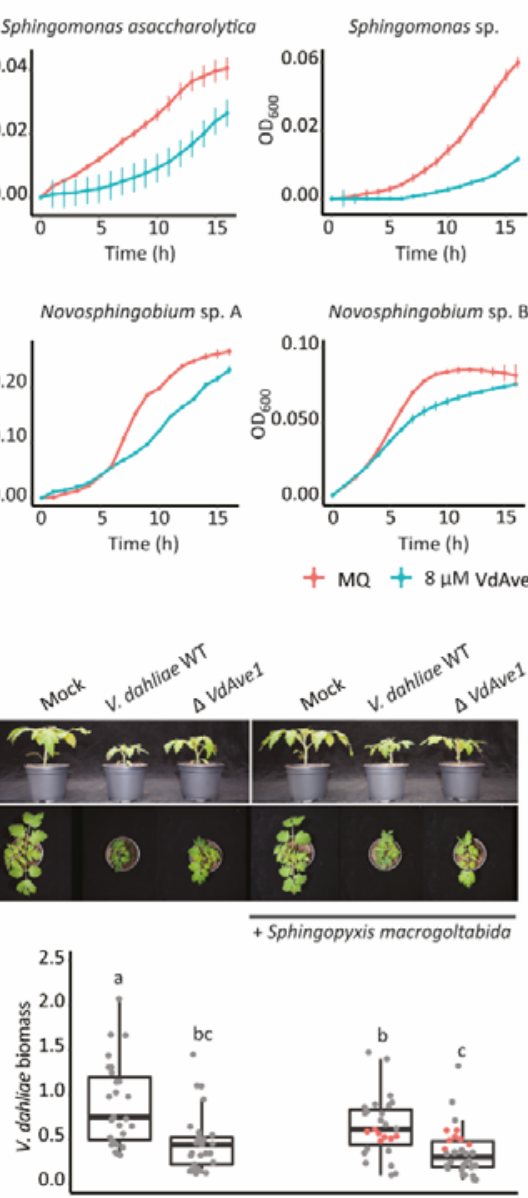

$+\mathrm{MQ}+8 \mu \mathrm{M}$ VdAve 1

e
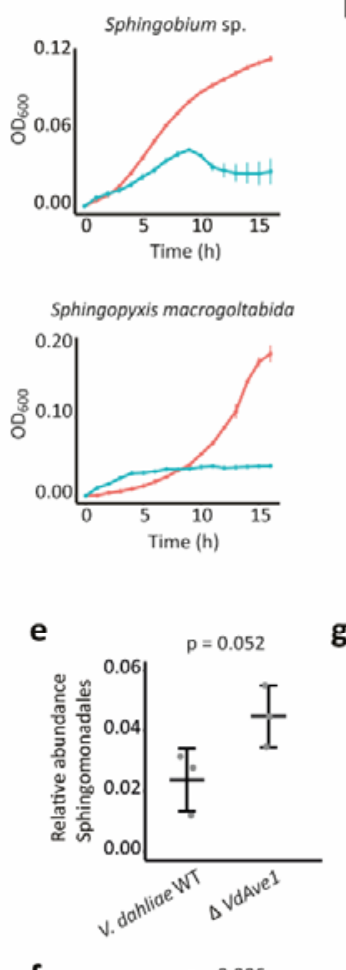

f

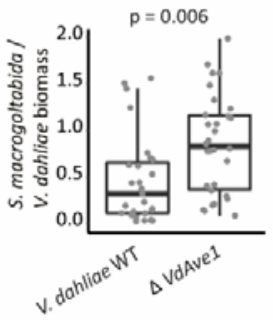

b
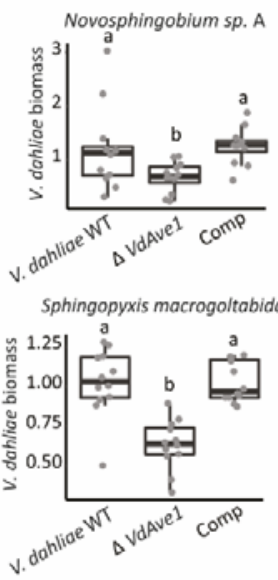

g

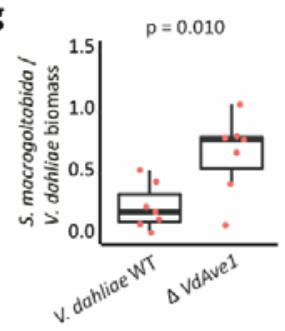

207

208

209

210

211

212

213

214

Fig. 3: Verticillium dahliae VdAve1 affects antagonistic Sphingomonads. a, Sphingomonads are inhibited by VdAve1 in tomato xylem fluid. $b, V d A v e 1$ supports $V$. dahliae growth in the presence of Sphingomonads. Biomass of wild-type V. dahliae (WT) and the VdAve1 deletion ( $\Delta V d A v e 1$ ) and complementation (Comp) mutants was quantified following 48 hours of co-cultivation with Sphingomonads in $0.5 \times$ MS medium ( $N=12$ ). Letters represent significant differences (one-way ANOVA and Tukey's post-hoc test; $\mathrm{p}<0.05$ for Novosphingobium sp.; $\mathrm{p}<0.0001$ for $S$. macrogoltabida). c, Tomato seed treatment with $S$. macrogoltabida reduces Verticillium wilt symptoms (stunting; 14 days post inoculation). d, V. dahliae biomass in tomato stems determined with real-time PCR. Letters 
represent significant biomass differences (one-way ANOVA and Tukey's post-hoc test; $p<0.05 ; \mathrm{N} \geq 27$ ). Each dot, grey or red, indicates the relative $V$. dahliae biomass in a single tomato plant. e, Relative abundance of Sphingomonadales according to $16 \mathrm{~S}$ ribosomal DNA profiling of tomato plants pretreated with S. macrogoltabida and infected with wild-type $V$. dahliae or the VdAve1 deletion mutant (unpaired student's t-test; $\mathrm{N}=3$ ). $\mathrm{f}$, Relative Sphingopyxis biomass in all pre-treated tomato plants infected with wild-type $V$. dahliae or the VdAve1 deletion mutant, indicated by the grey and red dots in Fig. $3 \mathrm{~d}$ combined, as quantified by real-time PCR (unpaired student's t-test; $\mathrm{N} \geq 27$ ). g, Relative Sphingopyxis biomass in pre-treated tomato plants colonized by similar amounts of wild-type $V$. dahliae or the VdAve1 deletion mutant, indicated by the red dots in Fig. $3 \mathrm{~d}$, as quantified by real-time PCR (unpaired student's t-test; $N=7$ ).

Our observation that $V$. dahliae secretes VdAve1 to suppress microbial competitors in the microbiomes of its hosts, prompted us to speculate about additional $V$. dahliae effector proteins involved in microbiome manipulation. Based on our findings that canonical effector genes such as VdAve1 can also be expressed outside the host, we hypothesized that $V$. dahliae also secretes effectors that aid in microbial competition in the soil. Therefore, to query for the occurrence of additional effectors that act in microbiome manipulation, the predicted secretome of $V$. dahliae strain JR2 ${ }^{26}$ was probed for structural homologs of known antimicrobial proteins (AMPs), revealing 10 candidates (Extended data Table 4). The majority of the identified effectors share typical characteristics with canonical host-targeting effector proteins, such as being small and rich in cysteines. However, based on previously performed RNA sequencing experiments, no expression of any of these candidates could be monitored during colonization of Arabidopsis thaliana, Nicotiana benthamiana or cotton plants (Extended data Fig. 6$)^{17,20,27,28}$. Additionally, in vitro cultivation of $V$. dahliae in the presence of E. coli, B. subtilis or T. viride, or of peptidoglycan to mimic bacterial encounter, did not lead to induction of any of the effector candidate genes (Extended data Fig. 6). Consequently, we hypothesized that these genes require other environmental triggers to be induced. Indeed, growth in soil extract consistently induced expression of candidate VdAMP2 (Fig. 4a) that shares structural homology (confidence $>90 \%$ ) with amphipathic $\beta$-hairpins of aerolysin-type $\beta$-pore forming toxins ( $\beta$-PFTs) (Extended data Fig. 7$)^{29}$. 


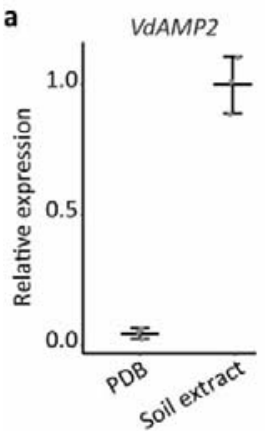

c
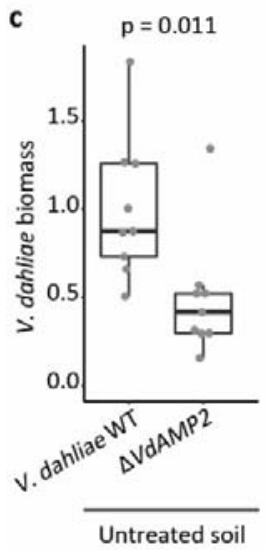

b
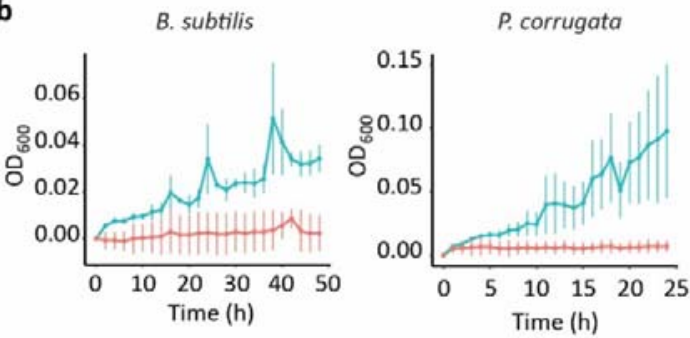

Culture filtrate: + V. dahliae WT $+p$ VdAve1::VdAMP2

d
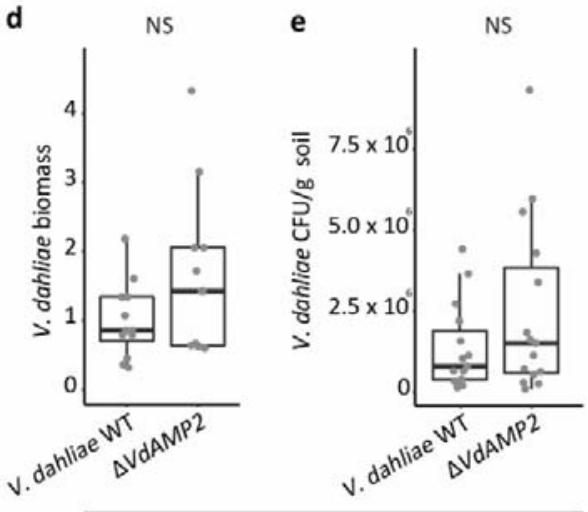

Sterilized soil

Fig. 4: VdAMP2 contributes to Verticillium dahliae soil colonization. a, V. dahliae VdAMP2 is induced after five days of cultivation in soil extract but not in potato dextrose broth (PDB). b, Growth of $B$. subtilis and $P$. corrugata in filter-sterilized culture filtrates from wild-type $V$. dahliae and the VdAMP2 expression transformant grown in liquid $0.2 x \mathrm{PDB}+0.5 \mathrm{x}$ MS medium. $\mathrm{c}$, VdAMP2 contributes to soil colonization. $V$. dahliae biomass in soil samples was determined by real-time PCR seven days after inoculation with wild-type $V$. dahliae (WT) and the VdAMP2 deletion mutant ( $\triangle V$ dAMP2) (unpaired student's t-test; $\mathrm{N}=9$ ). $\mathrm{d}$,e VdAMP2 does not contribute to colonization in sterile soil. Experiment as shown in $\mathbf{c}$ in sterile soil. $V$. dahliae biomass was quantified with real-time PCR (N=9), $d$, and by colony forming unit counts per gram of soil ( $N=15)$, e.

To test for potential antimicrobial activity of VdAMP2, we attempted heterologous production of the effector protein. However, since production in E. coli and Pichia pastoris repeatedly failed, production in V. dahliae under control of the VdAve1 promoter was pursued, resulting in high levels of VdAMP2 expression in vitro (Extended data Fig. 8a-c). Interestingly, proliferation of B. subtilis and of $P$. corrugata (Fig. 4b), but not of $F$. oxysporum and of $T$. viride (Extended data Fig. 9), was affected by filter-sterilized culture filtrate of the VdAMP2 expression transformant when compared with that of wild-type $V$. dahliae, suggesting that VdAMP2 exerts only antibacterial activity, like VdAve1 albeit with a different activity spectrum. Soil colonization assays using wild-type $V$. dahliae and a VdAMP2 
deletion mutant (Extended data Fig. 8d-f) demonstrated that VdAMP2 contributes to $V$. dahliae fitness in the soil as measured by biomass accumulation (Fig. 4c). Importantly, since this fitness contribution is not observed in sterilized soil, we conclude that VdAMP2 contributes to V. dahliae fitness through its efficacy in microbial competition (Fig. $4 \mathrm{~d}, \mathrm{e}$ ). As can be anticipated, the positive effect of VdAMP2 on biomass accumulation in the soil is reflected in disease development when plants are grown on this soil (Extended data Fig. 10), demonstrating that VdAMP2 positively contributes to virulence of $V$. dahliae in an indirect manner.

In conclusion, in this study we have demonstrated that $V$. dahliae employs effector proteins that contribute to niche colonization, during host-associated as well as during soil-dwelling stages, through the selective manipulation of local microbiomes. A wide array of microbially-secreted molecules has previously been described to fulfill crucial functions in intermicrobial competition, including hydrolytic enzymes, secondary metabolites and antimicrobial proteins. Some Gramnegative bacteria even employ a specialized type VI secretion system (T6SS) to translocate antimicrobial proteins into their microbial competitors ${ }^{30}$. In this manner, Vibrio cholerae, the causal agent of cholera, employs its T6SS to target members of the host commensal microbiota and hereby promotes colonization of the gut ${ }^{31}$. Similarly, the T6SS effector Hyde1 of the phytopathogen Acidovorax citrulli targets plant-associated bacteria in vitro and was speculated to play a role in microbial competition in planta ${ }^{7}$. This T6SS is analogous to the type III secretion system (T3SS) of Gram negative bacteria that acts as a needle-like structure to directly inject effector proteins into host cells to promote disease ${ }^{32}$. Similar secretion machinery intended for host-microbe or microbemicrobe interactions has not been described for fungi and other filamentous microbes, which instead secrete their effector proteins by extracellular deposition. Consequently, effector molecules targeted towards host cells or towards microbial competitors cannot be discriminated based on differential secretion motifs, such as those that determine type III versus type VI secretion in Gram negatives. Here, we have shown that the pool of effectors secreted by a fungal plant pathogen represents a diverse cocktail comprising proteins involved in the manipulation of the host as well as its 
microbiome. Consequently, the effectors reported here likely only represent a small proportion of a larger subset of the $V$. dahliae effector repertoire that is intended for microbiome manipulation. For instance, similar effectors might be crucial during advanced infection stages to prevent secondary infections by opportunistic microbes when host defenses are impaired. Additionally, effector proteins can be anticipated to facilitate the survival of the $V$. dahliae resting structures that persist in the microbe-rich soil for years ${ }^{33}$. After all, possibly, fungal effectors with host microbiomemanipulating capacity initially evolved to limit bacterial growth in soil, as the advent of fungi on earth preceded land plant evolution and fungi initially likely co-evolved with bacteria in soil to compete for organic carbon. The discovery of further molecules for microbiome manipulation secreted by $V$. dahliae and other microbes, and unravelling of underlying modes of action, may ultimately lead to the development of novel antibiotics. 


\section{Materials and methods}

All experiments have been repeated at least three times.

Xylem fluid isolation. Tomato plants (Solanum lycopersicum cv. Moneymaker) were grown under controlled greenhouse conditions as described previously ${ }^{34}$. The stems of six-week-old plants were cut to allow oozing of the xylem fluid, which was collected on ice with a vacuum pump. The collected xylem fluid was centrifuged for 10 minutes at $20000 \times \mathrm{g}$ and filter-sterilized using a $0.2 \mu \mathrm{m}$ filter (Sarstedt, Nümbrecht, Germany). The sterilized xylem fluid was stored at $-20^{\circ} \mathrm{C}$ until use.

Soil extract preparation. To prepare soil extract, 100 grams of dry potting soil (Lentse potgrond, substraat arabidopsis, Lentse Potgrond BV, Katwijk, the Netherlands) was mixed with $500 \mathrm{~mL}$ of demineralized water and autoclaved for 15 minutes at $121^{\circ} \mathrm{C}$. Soil particles were pelleted through centrifugation and the supernatant was collected and stored at $-20^{\circ} \mathrm{C}$ until use.

Gene expression analysis. Total RNA of $V$. dahliae strain JR2 was isolated from tomato roots seven days after root dip inoculation and following five days of in vitro growth in soil extract and potato dextrose broth (PDB) using the Maxwell ${ }^{\circledR} 16$ LEV Plant RNA Kit (Promega, Madison, USA). Real-time PCR was performed as described previously ${ }^{17}$ to determine the expression of effector genes relative to VdGAPDH with primer pairs as shown in Extended data Table 6.

Production and purification of recombinant effector proteins. The sequences encoding mature VdAve1 and VnAve1 were cloned into pET-15b with an $\mathrm{N}$-terminal His 6 tag sequence (Novagen, Madison, WI, USA) (primer sequences, see Extended data Table 6). The resulting expression vectors were confirmed by sequencing and used to transform E. coli strain BL21. For heterologous protein production, BL21 cells were grown in $1 \times \mathrm{YT}$ liquid medium at $37^{\circ} \mathrm{C}$ with constant shaking at $200 \mathrm{rpm}$. Protein production was induced with $1 \mathrm{mM}$ IPTG final concentration when cultures reached an $\mathrm{OD}_{600}=2$ to ensure maximum yields. Following 2 hours of protein production, the bacterial cells were pelleted and snap-frozen in liquid nitrogen and then washed with $100 \mathrm{mM} \mathrm{NaCl}, 1 \mathrm{mM}$ EDTA, and 10 
$\mathrm{mM}$ Tris at pH 8.5. Cells were disrupted by stirring for 1 hour in lysis buffer (100 mM Tris, $150 \mathrm{mM}$ $\mathrm{NaCl}, 10 \%$ glycerol, $6 \mathrm{mg} / \mathrm{mL}$ lysozyme (Sigma, St. Louis, MO, USA), $2 \mathrm{mg} / \mathrm{mL}$ deoxycholic acid, 0.06 $\mathrm{mg} / \mathrm{mL}$ DNasel, protease inhibitor cocktail (Roche, Mannheim, Germany)) at $4^{\circ} \mathrm{C}$. Soluble and insoluble fractions were separated by centrifuging at $20,000 \times \mathrm{g}$ for $10 \mathrm{~min}$. The insoluble protein pellets were washed with $10 \mathrm{~mL} 1 \mathrm{M}$ guanidine hydrochloride ( $\mathrm{GnHCl}$ ), $10 \mathrm{mM}$ Tris at $\mathrm{pH} 8.0$ and then denatured in $10 \mathrm{~mL} 6 \mathrm{M} \mathrm{GnHCl}, 10 \mathrm{mM} \beta$-mercaptoethanol, $10 \mathrm{mM}$ Tris at $\mathrm{pH}$ 8.0. Samples were incubated for 1 hour at room temperature. Non-denatured debris was pelleted by centrifuging at $20,000 \times g$ for $10 \mathrm{~min}$ and discarded. Denaturation was allowed to continue for additional 3-4 hours. Proteins were purified under denaturing conditions by metal affinity chromatography using a column packed with $50 \%$ His60 $\mathrm{Ni}^{2+}$ Superflow Resin (Clontech, Mountain View, CA, USA). The purified effector proteins were dialysed (Spectra/Por 3 Dialysis Membrane, MWCO=3.5 kDa) step-wise against 20 volumes of $0.25 \mathrm{M}$ ammonium sulfate, $0.1 \mathrm{M}$ BisTris, $10 \mathrm{mM}$ reduced glutathione, $2 \mathrm{mM}$ oxidized glutathione, pH 5.5 with decreasing $\mathrm{GnHCl}$ concentrations for refolding. Each dialysis step was allowed to proceed for at least 24 hours. Finally, proteins were dialysed against demineralized water. Final concentrations were determined using the BioRad Protein Assay (BioRad, Veenendaal, The Netherlands).

Functionality of refolded VdAve1 was confirmed through recognition by the corresponding tomato immune receptor Ve1. To this end, an overnight culture of $A$. tumefaciens strain GV3101 carrying the pSOL2092:Ve1 construct ${ }^{35}$ was harvested by centrifugation and re-suspended to $\mathrm{OD}_{600}=2$ in MMA (2\% sucrose, $0.5 \%$ Murashige \& Skoog salts (Duchefa Biochemie, Haarlem, The Netherlands), $10 \mathrm{mM}$ MES, $200 \mu \mathrm{M}$ acetosyringone, $\mathrm{pH}$ 5.6) and infiltrated in the leaves of 5-week-old N. tabacum (cv. Petite Havana SR1) plants. After 24 hours, $10 \mu \mathrm{M}$ of purified and refolded 6xHis-VdAve1 was infiltrated in leaf areas expressing Ve1. Photos were taken three days post infiltration of the effector protein. 
346 Generation of $V$. dahliae mutants. To generate the VdAMP2 effector deletion construct, VdAMP2

347 flanking sequences were amplified using the primers listed in Extended data Table 6 and cloned into $348 \mathrm{pRF}-\mathrm{HU} 2^{36}$. To allow expression of VdAMP2 under control of the VdAve1 promoter, the coding sequence of $V d A M P 2$ was amplified and cloned into pFBT005. All constructs were transformed into A. tumefaciens strain AGL1 for V. dahliae transformation as described previously ${ }^{37}$.

V. dahliae culture filtrates. Conidiospores of $V$. dahliae strain JR2 and the VdAMP2 expression transformant were harvested from potato dextrose agar (PDA) and diluted to a final concentration of $10^{4}$ conidiospores $/ \mathrm{mL}$ in $20 \mathrm{~mL}$ of $0.2 x$ PDB supplemented $+0.5 x$ Murashige \& Skoog medium (Duchefa, Haarlem, The Netherlands). Following four days of incubation at $22^{\circ} \mathrm{C}$ and $120 \mathrm{rpm}$, the fungal biomass was pelleted and the remaining supernatants were filter sterilized and stored at $-20^{\circ} \mathrm{C}$ until use.

Bacterial isolates. Bacterial strains Bacillus subtilis AC95, Staphylococcus xylosus M3, Pseudomonas corrugata C26, Streptomyces sp. NE-P-8 and Ralstonia sp. M21 were obtained from our in house endophyte culture collection. Strains used in this study were all isolated from the xylem vessels of tomato cultivars from commercial greenhouses, both from stem and leaf sections. All strains were identified based on their $16 \mathrm{~S}$ rRNA gene sequence using the primers $27 \mathrm{~F}$ and 1492R (Extended data Table 6). $16 \mathrm{~S}$ amplicons were sequenced by Sanger sequencing at Eurofins (Mix2Seq). The partial $16 \mathrm{~S}$ rRNA gene sequences obtained were evaluated against the $16 \mathrm{~S}$ ribosomal DNA sequence (Bacteria and Archaea) database from NCBI. Bacterial strains Acidovorax sp. (Leaf 73), Arthrobacter sp. (Leaf 69), Rhizobium sp. (Leaf 167), Serratia sp. (Leaf 50), Sphingomonas sp. (Leaf 198), Sphingobium sp. (Leaf 26) and Novosphingobium sp. B (Leaf 2) were obtained from the At-SPHERE collection ${ }^{25}$. Bacterial strains S. mali (DSM 10565) and S. asaccharolytica (DSM 10564) were obtained from the DSMZ culture collection (Braunschweig, Germany). Bacterial strains Novosphingobium sp. A (NCCB 100261), S. macrogoltabida (NCCB 95163), and Sphingobacterium sp. (NCCB 100093) were obtained from the Westerdijk Fungal Biodiversity Institute (Utrecht, The Netherlands). 
In vitro microbial growth assays. Bacterial isolates were grown on lysogeny broth agar (LBA) or tryptone soya agar (TSA) at $28^{\circ} \mathrm{C}$. Single colonies were selected and grown overnight at $28^{\circ} \mathrm{C}$ while shaking at $200 \mathrm{rpm}$. Overnight cultures were resuspended to $\mathrm{OD}_{600}=0.05$ in xylem fluid supplemented with purified effector proteins or diluted using culture filtrates to $\mathrm{OD}_{600}=0.1$. Additionally, $F$. oxysporum and $T$. viride spores were harvested from a PDA plate and suspended in xylem fluid supplemented with purified effector proteins or the $V$. dahliae culture filtrates to a final concentration of $10^{4}$ spores $/ \mathrm{mL}$. $200 \mu \mathrm{L}$ of the microbial suspensions was aliquoted in clear 96 well flat bottom polystyrene tissue culture plates. Plates were incubated in a CLARIOstar plate reader (BMG LABTECH, Ortenberg, Germany) at $22^{\circ} \mathrm{C}$ with double orbital shaking every 15 minutes (10 seconds at $300 \mathrm{rpm}$ ). The optical density was measured every 15 minutes at $600 \mathrm{~nm}$.

Scanning electron microscopy. Samples for scanning electron microscopy were prepared as described previously with slight modifications ${ }^{38}$. In short, B. subtilis strain AC95 was grown overnight in $L B$ and resuspended in xylem fluid to an $\mathrm{OD}_{600}=0.05$. Purified effector proteins were added to a final concentration of $6.5 \mu \mathrm{M}(=0.8 \times \mathrm{MIC}, \mathrm{VdAve} 1)$ and bacterial suspensions were incubated for 0 , 1,3 and 7 hours. Next, $20 \mu \mathrm{L}$ of the bacterial suspensions was transferred to poly-L-lysine coated glass slides (Corning, New York, USA) and incubated for another hour to allow binding of the bacteria. Glass slides were washed using sterile $M Q$ and samples were fixed using $2.5 \%$ glutaraldehyde followed by postfixation in $1 \%$ osmium tetroxide. Samples were dehydrated using an ethanol dehydration series and subjected to critical point drying using a Leica CPD300 (Leica Mikrosysteme GmbH, Vienna, Austria). Finally, the samples were mounted on stubs, coated with $12 \mathrm{~nm}$ of tungsten and visualized in a field emission scanning electron microscope (Magellan 400, FEI, Eindhoven, the Netherlands).

Root microbiome analysis. Tomato and cotton inoculations were performed as described previously ${ }^{34}$. After ten days, plants were carefully uprooted and gently shaken to remove loosely adhering soil from the roots. Next, roots with rhizosphere soil from three tomato or two cotton 
plants were pooled to form a single biological replicate. Samples were flash-frozen in liquid nitrogen and ground using mortar and pestle. Genomic DNA isolation was performed using the DNeasy PowerSoil Kit (Qiagen, Venlo, The Netherlands). Quality of the DNA samples was checked on a 1.0\% agarose gel. Sequence libraries were prepared following amplification of the V4 region of the bacterial $16 \mathrm{~S}$ rDNA ( $515 \mathrm{~F}$ and $806 \mathrm{R}$ ), and paired ends ( $250 \mathrm{bp}$ ) were sequenced using the HiSeq2500 sequencing platform (IIlumina, San Diego, USA) at the Beijing Genome Institute (BGI, Hong Kong, China).

Sequencing data was processed using $R$ version 3.3.2. as described previously ${ }^{39}$. Briefly, amplicon sequence variants (ASVs) were inferred from quality filtered reads (Phred score $>30$ ) using the DADA2 method ${ }^{40}$. Taxonomy was assigned using the Ribosomal Database Project training set (RDP, version 16) and mitochondria- and chloroplast-assigned ASVs were removed. Next, ASV frequencies were transformed according to library size to determine relative abundances. The phyloseq package (version 1.22.3) was used to determine $\alpha$-diversity (Shannon index) and $\beta$-diversity (Bray-Curtis dissimilarity) as described previously ${ }^{33,41}$. Differential abundance analysis was performed using the DESeq2 extension within phyloseq ${ }^{42}$. To this end, a parametric model was applied to the data and a negative binomial Wald test was used to test for differential abundance of bacterial taxa with $p<0.01$ as significance threshold.

In vitro competition assay. Conidiospores of $V$. dahliae strain JR2 and the VdAve1 deletion and complementation mutants were harvested from a PDA plate using sterile water and diluted to a final concentration of $10^{6}$ conidiospores/mL in liquid 0.5x MS (Murashige and Skoog) medium (Duchefa, Haarlem, The Netherlands). Next, overnight cultures of Novosphingobium sp. and S. macrogoltabida were added to the conidiospores to $\mathrm{OD}_{600}=0.05$ and $500 \mu \mathrm{L}$ of the microbial suspensions was aliquoted in clear 12-well flat-bottom polystyrene tissue culture plates. Following 48 hours of incubation at room temperature, the microbial cultures were recovered and genomic DNA was isolated using the SmartExtract - DNA Extraction Kit (Eurogentec, Maastricht, The Netherlands). $V$. 
dahliae biomass was quantified through real-time PCR using V. dahliae specific primers targeting the internal transcribed spacer (ITS) region of the ribosomal DNA (Extended data Table 6).

In planta competition assay. To allow S. macrogoltabida colonization of in the absence of other microbes, tomato seeds were incubated for five minutes in $2 \%$ sodium hypochlorite to ensure surface sterilization. Next, surface sterilized tomato seeds were washed three times using sterile water and transferred to a sterile Petri dish containing a filter paper pre-moistened with a $S$. macrogoltabida suspension in water $\left(\mathrm{OD}_{600}=0.05\right)$. The tomato seeds were allowed to germinate in vitro and eventually transferred to regular potting soil, ten-day-old seedlings were inoculated as described previously ${ }^{34}$. Tomato stems were collected at 14 days post inoculation (dpi) and lyophilized prior to genomic DNA isolation with a CTAB-based extraction buffer (100 mM Tris- $\mathrm{HCl}$ pH 8.0, $20 \mathrm{mM}$ EDTA, $2 \mathrm{M} \mathrm{NaCl}, 3 \% \mathrm{CTAB})$. V. dahliae biomass was quantified with real-time PCR on the genomic DNA by targeting the internal transcribed spacer (ITS) region of the ribosomal DNA. The tomato rubisco gene was used for sample calibration. S. macrogoltabida biomass was quantified using Sphingopyxis specific primers (Extended data Table 6) and normalized using the V. dahliae ITS. Additionally, the relative abundance of the Sphingomonadales in three representative samples was determined by 165 ribosomal DNA profiling as described previously.

Soil colonization assays. Conidiospores of the V. dahliae strain JR2 and the mutants were harvested from PDA plate and a total of $10^{6}$ conidiospores were added to 1 gram of potting soil. Samples were incubated at room temperature in the dark. After one week, DNA was extracted from the soil samples using the DNeasy PowerSoil Kit (QIAGEN, Venlo, The Netherlands). V. dahliae biomass was quantified through real-time PCR using $V$. dahliae specific primers targeting the internal transcribed spacer (ITS) region of the ribosomal DNA (Extended data Table 6). Primers targeting a conserved region of the bacterial 165 rRNA gene were used for sample equilibration.

To allow sample calibration when using sterilized potting soil (15 minutes at $121^{\circ} \mathrm{C}$ ), the samples were first mixed in a $1: 1$ ratio with fresh potting soil prior to DNA extraction. 
446 Additionally, after one week of incubation of $V$. dahliae in the sterilized soil, serial dilutions were

447 made and plated onto PDA to quantify colony forming units.

448 Disease assays using $V$. dahliae microsclerotia. $V$. dahliae microsclerotia were produced in a sterile

449 moist medium of vermiculite and maize meal as described previously ${ }^{43}$. After four weeks of

450 incubation, the vermiculite/microsclerotia mixture was dried at room temperature. Next, $150 \mathrm{~mL}$ of

451 the dried mixture was mixed with $1 \mathrm{~L}$ of potting soil (Lentse potgrond, substraat arabidopsis, Lentse

452 Potgrond BV, Katwijk The Netherlands) and Arabidopsis seeds of the Col-0 ecotype were sown at

453 equal distances on top of the mixture. The above-ground parts of the plants were collected at $27 \mathrm{dpi}$

454 and $V$. dahliae biomass was quantified through real-time PCR using $V$. dahliae specific primers

455 targeting the internal transcribed spacer (ITS) region of the ribosomal DNA. The Arabidopsis rubisco

456 gene was used for sample calibration (Extended data Table 6). 


\begin{tabular}{|c|c|c|}
\hline 457 & $\operatorname{Re}$ & \\
\hline 458 & 1 & Rovenich, H., Boshoven, J. C. \& Thomma, B. P. H. J. Filamentous pathogen effector functions: \\
\hline 459 & & of pathogens, hosts and microbiomes. Current opinion in plant biology 20, 96-103 (2014). \\
\hline 460 & 2 & Stergiopoulos, I. \& de Wit, P. J. Fungal effector proteins. Annual Review of Phytopathology \\
\hline 461 & & 47, 233-263 (2009). \\
\hline 462 & 3 & Huttenhower, C. et al. Structure, function and diversity of the healthy human microbiome. \\
\hline 463 & & Nature $486,207-214$ (2012). \\
\hline 464 & 4 & Turner, T. R., James, E. K. \& Poole, P. S. The plant microbiome. Genome Biology 14, 209 \\
\hline 465 & & (2013). \\
\hline 466 & 5 & Bulgarelli, D. et al. Revealing structure and assembly cues for Arabidopsis root-inhabiting \\
\hline 467 & & bacterial microbiota. Nature 488 91-95 2012). \\
\hline 468 & 6 & Lundberg, D. S. et al. Defining the core Arabidopsis thaliana root microbiome. Nature 488, \\
\hline 469 & & $86-90(2012)$ \\
\hline 470 & 7 & Levy, A. et al. Genomic features of bacterial adaptation to plants. Nature Genetics $50,138-$ \\
\hline 471 & & 150 (2017). \\
\hline 472 & 8 & Koprivova, A. et al. Root-specific camalexin biosynthesis controls the plant growth-promoting \\
\hline 473 & & effects of multiple bacterial strains. PNAS 116 (2019). \\
\hline 474 & 9 & Huang, A. C. et al. A specialized metabolic network selectively modulates Arabidopsis root \\
\hline 475 & & microbiota. Science 364 (2019). \\
\hline 476 & 10 & Rudrappa, T., Czymmek, K. J., Pare, P. W. \& Bais, H. P. Root-secreted malic acid recruits \\
\hline 477 & & beneficial soil bacteria. Plant physiology 148, 1547-1556 (2008). \\
\hline 478 & 11 & Berendsen, R. L., Pieterse, C. M. \& Bakker, P. A. The rhizosphere microbiome and plant \\
\hline 479 & & health. Trends in plant science 17, 478-486 (2012). \\
\hline 480 & 12 & Berendsen, R. L. Disease-induced assemblage of a plant-beneficial bacterial consortium. The \\
\hline 481 & & ISME journal, 12, 1496- 1507 (2018) \\
\hline 482 & 13 & Snelders, N. C., Kettles, G. J., Rudd, J. J. \& Thomma, B. P. H. J. Plant pathogen effector \\
\hline 483 & & proteins as manipulators of host microbiomes? Molecular plant pathology 19, 257-259 \\
\hline 484 & & (2018). \\
\hline 485 & 14 & Fradin, E. F. \& Thomma, B. P. H. J. Physiology and molecular aspects of Verticillium Wilt \\
\hline 486 & & diseases caused by V. dahliae and V. albo-atrum. Molecular plant pathology 7, 71-86 (2006). \\
\hline 487 & 15 & Klosterman, S. J., Atallah, Z. K., Vallad, G. E. \& Subbarao, K. V. Diversity, pathogenicity, and \\
\hline 488 & & management of Verticillium species. Annual Review of Phytopathology 47, 39-62 (2009). \\
\hline 489 & 16 & Mol, L. \& Van Riessen, H. Effect of plant roots on the germination of microsclerotia of \\
\hline 490 & & Verticillum dahliae. European journal of plant pathology 101, 673-678 (1995). \\
\hline 491 & 17 & de Jonge, R. et al. Tomato immune receptor Ve1 recognizes effector of multiple fungal \\
\hline 492 & & pathogens uncovered by genome and RNA sequencing. PNAS 109, 5110-5115 (2012). \\
\hline 493 & 18 & Ficarra, F. A., Grandellis, C., Garavaglia, B. S., Gottig, N. \& Ottado, J. Bacterial and plant \\
\hline 494 & & natriuretic peptides improve plant defence responses against pathogens. Molecular plant \\
\hline 495 & & pathology 19, 801-811 (2018). \\
\hline 496 & 19 & Gehring, C. A. \& Irving, H. R. Natriuretic peptides - a class of heterologous molecules in \\
\hline 497 & & plants. International Journal of Biochemistry \& Cell Biology 35, 1318-1322 (2003). \\
\hline 498 & 20 & Faino, L., de Jonge, R. \& Thomma, B. P. H. J. The transcriptome of Verticillium dahliae- \\
\hline 499 & & infected Nicotiana benthamiana determined by deep RNA sequencing. Plant Signaling \& \\
\hline 500 & & Behavior 7, 1065-1069 (2012). \\
\hline 501 & 21 & Ingham, A. B. \& Moore, R. J. Recombinant production of antimicrobial peptides in \\
\hline 502 & & heterologous microbial systems. Biotechnology and Applied Biochemistry 47, 1-9 (2007). \\
\hline 503 & 22 & Takeuchi, M., Hamana, K. \& Hiraishi, A. Proposal of the genus Sphingomonas sensu stricto \\
\hline 504 & & three new genera, Sphingobium, Novosphingobium and Sphingopyxis, on the basis \\
\hline
\end{tabular}


phylogenetic and chemotaxonomic analyses. International Journal of Systematic and Evolutionary Microbiology 51, 1405-1417 (2001).

23 Aylward, F. O. et al. Comparison of 26 Sphingomonad genomes reveals diverse environmental adaptations and biodegradative capabilities. Applied and Environmental Microbiology 79, 3724-3733 (2013).

24 Innerebner, G., Knief, C. \& Vorholt, J. A. Protection of Arabidopsis thaliana against leafpathogenic Pseudomonas syringae by Sphingomonas strains in a controlled model system. Applied and Environmental Microbiology 77, 3202-3210 (2011).

25 Bai, Y. et al. Functional overlap of the Arabidopsis leaf and root microbiota. Nature 528, 364369 (2015).

26 Faino, L. et al. Single-molecule real-time sequencing combined with optical mapping yields completely finished fungal genome. mBio 6 (2015).

27 Depotter, J. R. L. et al. Homogenization of sub-genome secretome gene expression patterns in the allodiploid fungus Verticillium longisporum. Preprint at https://www.biorxiv.org/content/10.1101/341636v1 (2018).

28 Gibriel, H., Li, J., Zhu, L., Seidl, M. \& Thomma, B. P. H. J. Verticillium dahliae strains that infect the same host plant display highly divergent effector catalogs. Preprint at https://www.biorxiv.org/content/10.1101/528729v1 (2019).

29 Dal Peraro, M. \& van der Goot, F. G. Pore-forming toxins: ancient, but never really out of fashion. Nature Reviews Microbiology 14, 77-92 (2016).

30 Coulthurst, S. The Type VI secretion system: a versatile bacterial weapon. Microbiology 165, 503-515 (2019).

31 Zhao, W., Caro, F., Robins, W. \& Mekalanos, J. J. Antagonism toward the intestinal microbiota and its effect on Vibrio cholerae virulence. Science 359, 210-213 (2018).

32 Alfano, J. R. \& Collmer, A. Type III secretion system effector proteins: double agents in bacterial disease and plant defense. Annual Review of Phytopathology 42, 385-414 (2004).

33 Xiong, D. et al. Deep mRNA sequencing reveals stage-specific transcriptome alterations during microsclerotia development in the smoke tree vascular wilt pathogen, Verticillium dahliae. BMC Genomics 15, 324, (2014).

34 Fradin, E. F. et al. Genetic dissection of Verticillium Wilt resistance mediated by tomato Ve1. Plant physiology 150, 320-332 (2009).

35 Zhang, Z. et al. Optimized agroinfiltration and virus-induced gene silencing to study Ve1mediated Verticillium resistance in tobacco. Molecular Plant Microbe Interactions 26, 182190, (2013).

36 Frandsen, R. J., Andersson, J. A., Kristensen, M. B. \& Giese, H. Efficient four fragment cloning for the construction of vectors for targeted gene replacement in filamentous fungi. $B M C$ Molecular Biology 9, 70 (2008).

37 Santhanam, P. in Plant Fungal Pathogens 509-517 (Springer, 2012).

38 Bozzola, J. J. Conventional specimen preparation techniques for scanning electron microscopy of biological specimens. Methods in Molecular Biology 1117, 133-150 (2014).

39 Callahan, B. J., Sankaran, K., Fukuyama, J. A., McMurdie, P. J. \& Holmes, S. P. Bioconductor Workflow for Microbiome Data Analysis: from raw reads to community analyses. F1000Research 5, 1492 (2016).

40 Callahan, B. J. et al. DADA2: High-resolution sample inference from Illumina amplicon data. Nature Methods 13, 581, (2016).

41 McMurdie, P. J. \& Holmes, S. phyloseq: an R package for reproducible interactive analysis and graphics of microbiome census data. Plos One 8, e61217 (2013).

42 Love, M. I., Huber, W. \& Anders, S. Moderated estimation of fold change and dispersion for RNA-seq data with DESeq2. Genome Biology 15, (2014). 
43 Depotter, J. R. L., Thomma, B. P. H. J. \& Wood, T. A. Measuring the impact of Verticillium longisporum on oilseed rape (Brassica napus) yield in field trials in the United Kingdom. European Journal of Plant Pathology 153, 321-326 (2019).

44 de Jonge, R. et al. Extensive chromosomal reshuffling drives evolution of virulence in an asexual pathogen. Genome Research 23, 1271-1282 (2013).

45 Kelley, L. A. et al. The Phyre2 web portal for protein modeling, prediction and analysis. Nature protocols, 10, 845-858 (2015)

Acknowledgments: The authors thank M. Giesbers from the Wageningen Electron Microscopy Centre for technical assistance. Work in the laboratory of B.P.H.J.T is supported by the Research Council Earth and Life Sciences (ALW) of the Netherlands Organization of Scientific Research (NWO).

Author contributions: N.C.S., H.R. and B.P.H.J.T. conceived the project. N.C.S., H.R., G.C.P., J.R.M. and B.P.H.J.T. designed the experiments. N.C.S., H.R., G.C.P., M.R.F., M.F.S. and R.N. carried out the experiments, N.C.S., H.R., G.C.P., M.R.F., M.F.S., J.A.V., R.N., J.R.M. and B.P.H.J.T. analysed the data. N.C.S. and B.P.H.J.T. wrote the manuscript. All authors read and approved the final manuscript.

Competing interests: Authors declare no competing interests.

Data and materials availability: The metagenomics data have been deposited in the European Nucleotide Archive (ENA) under accession number PRJEB34281. 